\title{
Collagen of umbilical cord vein and its alterations in pre-eclampsia
}

\author{
Lech Romanowicz $^{1 凶}$ and Stefan Jaworski ${ }^{2}$ \\ ${ }^{1}$ Department of Medical Biochemistry, and ${ }^{2}$ Department of Gynecology, Medical Academy of \\ Biatystok, Biatystok, Poland
}

Received: 25 July, 2001; revised: 24 April, 2002; accepted 10 May, 2002

Key words: collagen, pre-eclampsia, umbilical cord vein

\begin{abstract}
The state of the vascular system of the mother and of placenta is known to exert a great influence on intrauterinal development of the fetus. Pre-eclampsia is the most common pathological syndrome connected with pregnancy. Since collagen is one of the main constituents of the vessel wall a comparison was made with collagen content and its molecular polymorphism in umbilical cord veins of newborns from healthy and pre-eclamptic mothers. It was found that umbilical cord veins of newborns from mothers with pre-eclampsia contained $18 \%$ less collagen than those of the newborns from normal pregnancies. This decrease was accompanied by a slight decrease of collagen solubility, but all its types (I, III, IV, V and VI) were present. However, the umbilical vein wall of newborns from mothers with pre-eclampsia contained relatively less of type I and more of type III collagen than the normal umbilical cord. These differences may be connected with a disturbance of blood flow in fetus of a woman with pre-eclampsia.
\end{abstract}

It is well known that the state of the vascular system of the mother and of placenta exerts an important influence on intrauterinal development of fetus (Baker et al., 1989; Fox, 1997; Howard, 1987). Pre-eclampsia is the most common pathological syndrome connected with pregnancy (Rippmann, 1981). It is accompanied by significant morphological changes in the vessel walls of the uterus and placenta.
Contraction of small arteries, hypertrophia of media, edema of intima and degenerative changes in arterial walls have been observed (Brosens, 1977; Cretti \& Jaworski, 1976; Kitzmiller \& Bernirschke, 1973). Omental arteries of women with pre-eclampsia have a significantly smaller internal diameter than the arteries of normotensive pregnant women (Aalkjaer et al., 1985).

\footnotetext{
${ }^{\square}$ Correspondence should be addressed to: Lech Romanowicz, Department of Medical Biochemistry, Medical Academy of Białystok, A. Mickiewicza 2, 15-230 Białystok 8, Poland; phone: (48 85) 742 4781; fax: (48 85) 742 4907; e-mail: zdbioch@amb.ac.bialystok.pl
}

Abbreviation: UCA, umbilical cord artery. 
Collagen is a group of at least 19 genetically distinct proteins (Van der Rest \& Garrone, 1991). The venous walls contain mainly type I, III, IV and V collagens (Barnes, 1983; Gilligan \& Spector, 1984). It is well known that both collagen content and the relationship between collagens of various types change in some pathological conditions (Barnes, 1983; 1985; Gilligan \& Spector, 1984). Previously we have compared the collagen content, its solubility and molecular polymorphism in normal umbilical cord arteries and Wharton's jelly with that from newborns from mothers with pre-eclampsia (Bańkowski, 1999; Bańkowski et al., 1993; 1996).

In the present paper we have examined umbilical cord veins to obtain more information on the possible significance of collagen constituents of umbilical vessel walls from pathologic pregnancies.

\section{MATERIAL AND METHODS}

Tissue material. Studies were performed on umbilical cord veins taken from 10 control and 10 pre-eclamptic newborns. Both the control and the pre-eclamptic babies were born between the 38 th and 41st week of gestation by physiological delivery or by cesarean section.

The control material was taken from newborns delivered by healthy mothers, aged 18-35 with normal blood pressure (systolic 100-139 mm Hg, diastolic 70-89 mm Hg), without any symptoms of edema or renal failure. The mean body mass of newborns was $3720 \pm 428 \mathrm{~g}$.

Mothers with pre-eclampsia demonstrated elevated blood pressure (systolic $>140 \mathrm{~mm}$ $\mathrm{Hg}$, diastolic $>90 \mathrm{~mm} \mathrm{Hg}$ ), detectable proteinuria, and edema after bed rest. The mean body mass of these newborns was $3235 \pm$ $610 \mathrm{~g}$.

Immediately after delivery, about $20 \mathrm{~cm}$ long sections of the umbilical cords were excised beginning from their placental end. The sam- ples of veins were carefully separated from the adjacent tissues, cut into small pieces, washed with physiological saline and submitted to biochemical studies.

Collagen content. The samples of veins taken from 10 newborns of either group were suspended in anhydrous acetone, homogenized with a knife homogenizer and dehydrated in the same solvent $\left(4^{\circ} \mathrm{C}, 48 \mathrm{~h}\right)$. The homogenate was defatted by extraction with the mixtures of acetone:ether $(1: 1, \mathrm{v} / \mathrm{v})$ and methanol:chloroform $(1: 1, \mathrm{v} / \mathrm{v})$ at room temperature for $48 \mathrm{~h}$. The defatted material was dried at room temperature to a constant weight. Collagen content was measured by the assay of hydroxyproline as described by Woessner (1976). Since hydroxyproline constitutes about $1 / 8(\mathrm{w} / \mathrm{w})$ of collagen weight, the approximate amount of this protein in arterial tissue was calculated by multiplication of hydroxyproline content by 8 .

Collagen solubility assay. The samples of veins taken from 10 newborns of either group were combined, cut into small pieces, divided into 4 parts, suspended in $1 \mathrm{M} \mathrm{NaCl}$ and homogenized with a knife homogenizer at $0-4^{\circ} \mathrm{C}$. The homogenates were extracted with the same solution at $4^{\circ} \mathrm{C}$ for $24 \mathrm{~h}$ with continuous stirring and then centrifuged at $25000 \times$ $g$ for $30 \mathrm{~min}$. The supernatants were collected, the sediments were extracted with the same solution and reextracted (usually 4 times) until a negative biuret reaction. The remnants after the last extraction (with $1 \mathrm{M} \mathrm{NaCl}$ ) were extracted with $0.15 \mathrm{M}$ citrate, $\mathrm{pH} 3.7$, and the combined supernatants centrifuged as above (Schofield et al., 1971). The remaining insoluble sediments were depolymerized in $4 \%$ EDTA as described by Steven (1967), centrifuged off and the remnants were extracted with $0.2 \mathrm{M}$ acetic acid at $4^{\circ} \mathrm{C}$ (Steven, 1967). The quantities of hydroxyproline in all supernatants and insoluble residues were determined.

Fractionation of collagen. The veins taken from 10 newborns of either group were combined, cut into small pieces, divided into 4 
parts and homogenized in $0.2 \mathrm{M}$ acetic acid with the use of a knife homogenizer at $4^{\circ} \mathrm{C}$. The homogenates digested with pepsin $(0.5$ $\mathrm{mg} / \mathrm{ml}$ ) at $4^{\circ} \mathrm{C}$ for $24 \mathrm{~h}$ with continuous stirring were centrifuged at $25000 \times \boldsymbol{g}$ for 30 min. The digestion procedure was repeated three times in the same conditions to hydrolyze noncollagenous proteins. Total collagen soluble in $0.5 \mathrm{M}$ acetic acid (Chung \& Miller, 1974) was precipitated with $0.7 \mathrm{M} \mathrm{NaCl}$ and fractionated into I-VI collagen types as described by Murata et al. (1986) using successive extractions with $\mathrm{NaCl}$ of increasing molarity at different $\mathrm{pH}$ values.

Collagen chain fragmentation with $\mathrm{CNBr}$. Both protein digest and insoluble sediment were dialyzed against $0.02 \mathrm{M} \mathrm{Na}_{2} \mathrm{HPO}_{4}$, $\mathrm{pH} 8.2$, at $4^{\circ} \mathrm{C}$. After centrifugation $70 \%$ formic acid was added to the precipitate. $\mathrm{CNBr}$ treatment was performed as described by Murata et al. (1986). The amounts of $\mathrm{CNBr}$ peptide bands of $\alpha$-chains were densitometrically determined for type I and type III collagen and calculated by the formula reported by Murata et al. (1986).

Western blot analysis. Supernatants and precipitates after repeated pepsin digestion of tissue homogenates, and fractions of the solubilized collagen were submitted to electrophoresis on sodium dodecyl sulphate/10\% polyacrylamide gel (SDS/PAGE) as described by Laemmli (1970). Since types III, IV and VI collagens contain disulphide bonds between their subunits these samples were submitted additionally to reduction with dithiothreitol. After SDS/PAGE the gels were allowed to equilibrate in $0.025 \mathrm{M}$ Tris/0.2 $\mathrm{M}$ glycine in $20 \%(\mathrm{v} / \mathrm{v}$ ) methanol for $5 \mathrm{~min}$. The protein was transferred to $0.2 \mu \mathrm{m}$ pore-sized nitrocellulose at $100 \mathrm{~mA}$ for $1 \mathrm{~h}$ by using a SigmaAldrich SU20-SDB unit. Blocking of collagen was made with $1 \%$ BSA in TBS-T (20 mM Tris/HCl, pH 7.4, containing $150 \mathrm{mM} \mathrm{NaCl}$ and $0.05 \%$ Tween 20 ) for $1 \mathrm{~h}$ at room temperature, washed three times in TBS-T and submitted to antibody binding by incubating with either anti-human collagen type I, III, IV, V or
VI mouse monoclonal antibody (ICN Biomedicals, Inc.) at 1:1000 in 5\% dried, defatted milk in $10 \mathrm{ml}$ TBS-T ( $4^{\circ} \mathrm{C}$, overnight). The membrane was washed three times with TBS-T, and, to detect collagen, alkaline phosphatase conjugated antibody goat anti-mouse IgG (whole molecule) was added at a concentration of 1:7500 in TBS-T and incubated for 1 $\mathrm{h}$ at room temperature. After four washings with TBS-T the membrane was submitted to Sigma-Fast BCIP/NBT reagent.

Statistical analysis. Mean values from 10 assays \pm standard deviations (S.D.) were calculated. Statistical analysis was performed using Student's $t$-test, accepting $P<0.05$ as significant.

\section{RESULTS}

The walls of control umbilical cord veins contained about $390 \mathrm{mg}$ of collagen per gram of dry mass, whereas those taken from pre-eclamptic newborns contained 18\% significantly less of this protein (Fig. 1A).

Solubility of collagen from umbilical veins was very low: only about $6 \%$ of total collagen both from control and pre-eclamptic veins (Fig. 1B) was extracted with $1 \mathrm{M} \mathrm{NaCl}$ and $0.15 \mathrm{M}$ citrate ( $\mathrm{pH}$ 3.7). A part of polymeric (insoluble) collagen was depolymerized with $4 \%$ EDTA and solubilized in $0.2 \mathrm{M}$ acetic acid. As can be seen from Fig. 1B the collagen of pre-eclamptic veins was less soluble in EDTA and acetic acid (1.4\%) than collagen of control veins $(1.9 \%)$.

During digestion of vein homogenates with pepsin in acetic acid collagen was solubilized, and fractionated to yielded five collagen fractions. For identification of particular collagen type each collagen fraction was then submitted to SDS/PAGE and for Western blot analysis the respective anti-human collagen mouse monoclonal antibodies were used. Comparison of the data presented in this paper with those presented by Murata et al. (1986) and described in our earlier studies (Bańkowski et 
$\mathrm{A}$

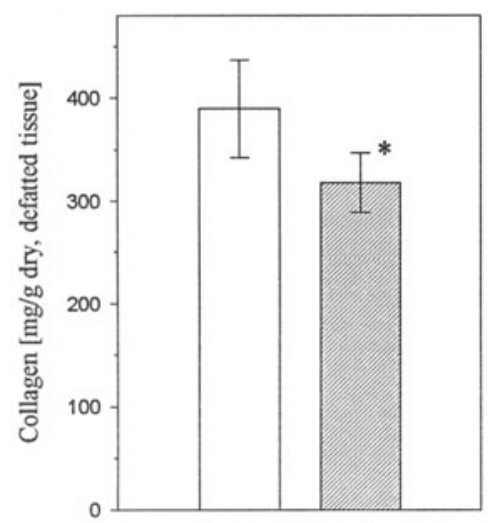

B

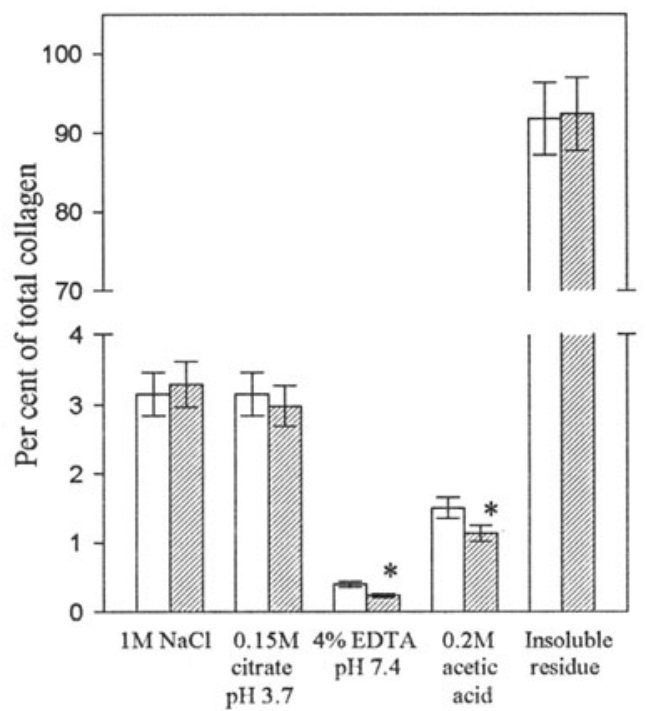

Figure 1. The content (A) and solubility (B) of collagen in the walls of control and pre-eclamptic veins.

Mean values from 10 (content) or 4 (solubility) assays \pm S.D.; $(\square)$, control veins; $(\square)$, pre-eclamptic veins, ${ }^{*} P<$ 0.001 .

$a l ., 1993$; 1996) indicate that umbilical vein contains all typical collagen subunits type: I, III, IV, V and VI. Figure 2A shows that the anti-human collagen type I monoclonal antibody reacted with one $\alpha$-subunit detected directly in pepsin digests of both control (lane 1) and pre-eclamptic (lane 2) veins and corresponds to the type I collagen fraction obtained by the method of Murata et al. (1986) (lanes 5 and 6). Figure 2B presents a typical electrophoretic pattern of type III collagen. Since $\alpha_{1}$ (III) subunit penetrates the gel only after reduction of disulphide bonds (lane 3 and 4 for pepsin digests, and lanes 7 and 8 for the fractions obtained according to Murata et al. (1986)).

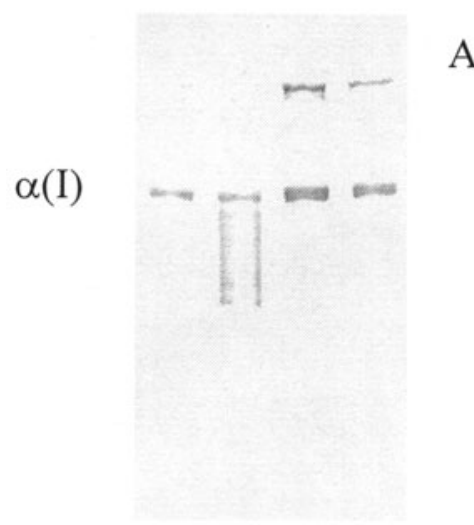

A

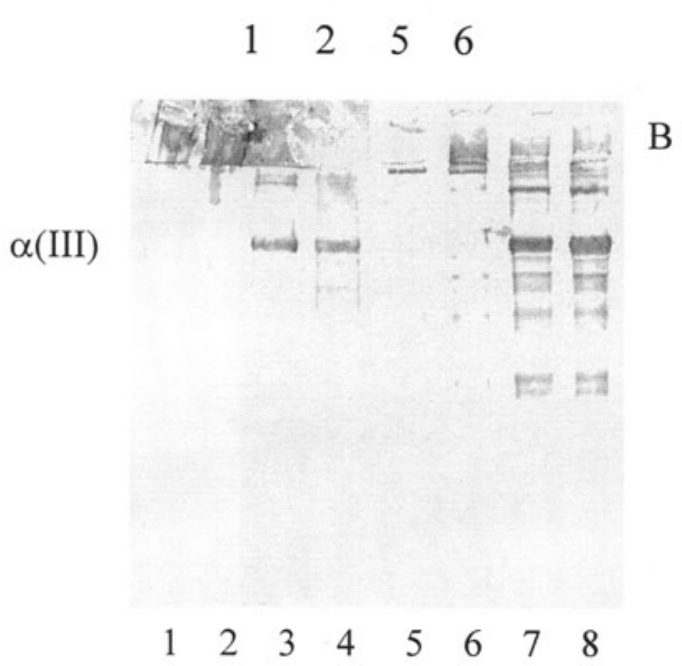

Figure 2. Western immunoblot analysis of collagen type: I (A) and III (B) in umbilical cord veins using specific antibody against each collagen type.

The position of $\alpha$ subunit is marked on the left. In type III collagen disulphide bonds have been reduced with dithiothreitol. Lane 1, pepsin digest of control vein; lane 2, pepsin digest of pre-eclamptic vein; lane 3 , as 1 treated with dithiothreitol; lane 4 , as 2 treated with dithiothreitol; lane 5 , the collagen fraction isolated from control vein by the differential salt precipitation method of Murata et al. (1986); lane 6, the collagen fraction isolated from pre-eclamptic vein by the differential salt precipitation method of Murata et al. (1986); lane 7 , as 5 treated with dithiothreitol; lane 8 , as 6 treated with dithiothreitol.

$\mathrm{CNBr}$ treatment of pepsin-digested vein homogenates allowed to evaluate the relationship between collagens type I and type III (Fig. 3). In the control vein the ratio of collagen type I to collagen type III amounted 3:1. This ratio changed to about $2: 1$ in the preeclamptic vein. Such pre-eclampsia-associated alterations in the type I/type III collagen ratio 


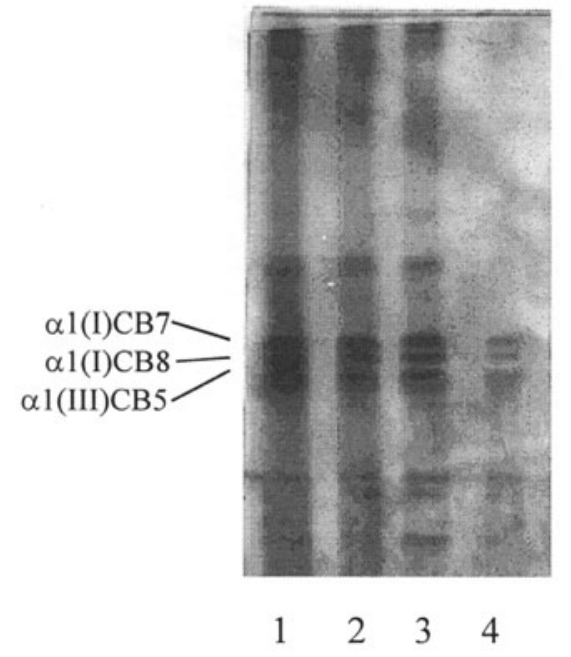

Figure 3. Electrophoretic mobilities of CNBr-peptides following degradation of the pepsin treated vein.

Lane 1, pepsin digest of control vein; lane 2, pepsin digest of pre-eclamptic vein; lane 3 , the remnant following pepsin digestion of control vein; lane 4 , the remnant following pepsin digestion of pre-eclamptic vein.

were found both in supernatants and sediments. Figure 3 shows electrophoregram of $\mathrm{CNBr}$ treated supernatants and sediments of control and pre-eclamptic veins after pepsin digestion in acetic acid.

Type I and type III collagens were found to be the most abundant in the umbilical cord vein collagens. In the control veins they constituted about $70.0 \%$ and $23.3 \%$ of total collagen, respectively. The amounts of type IV, V and VI collagens were distinctly lower. They constituted $1.0 \%, 2.8 \%$ and $3.0 \%$ of total collagen, respectively. The walls of pre-eclamptic veins contained a significantly higher percentage of type III collagen (28.8\%) than the control veins. At the same time the proportion of type I collagen was distinctly lowered reaching the value of $63.9 \%$. The amounts of collagen type IV, V and VI were unchanged (Fig. 4).

\section{DISCUSSION}

A rearrangement of extracellular matrix takes place in the walls of umbilical cord veins of pre-eclamptic newborns not observed in controls. First of all we have found that control veins contain about $390 \mathrm{mg}$ of collagen per gram of dry tissue while in pre-eclamptic veins collagen was lower by $18 \%$. In contrast, the pre-eclamptic umbilical cord artery (UCA) contained more than twice as much of that protein in comparison to control UCA wall (Bańkowski et al., 1993). Collagen content in Wharton's jelly was almost twice as high as in

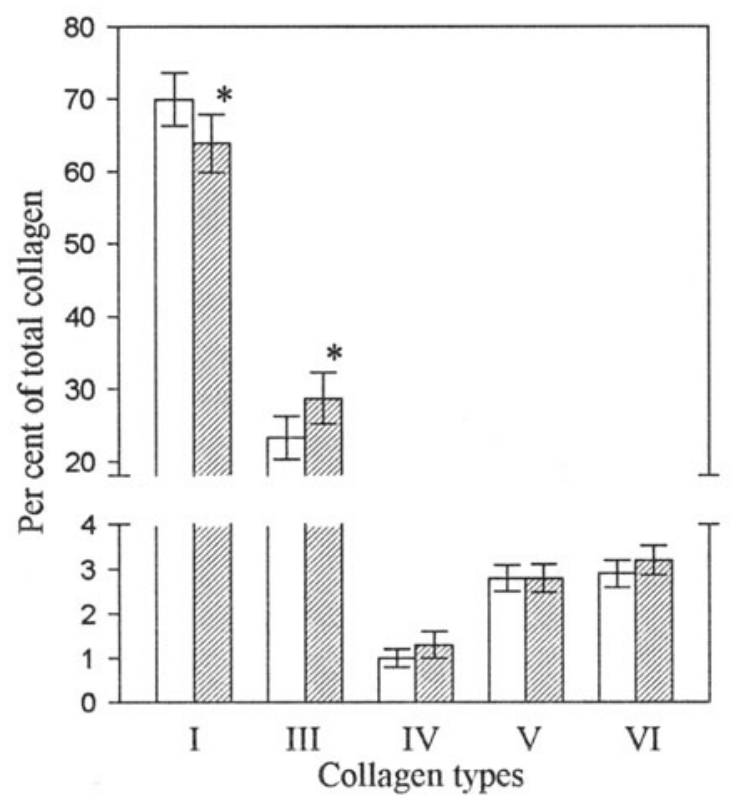

Figure 4. Percentage of different collagen types in the walls of control and pre-eclamptic veins.

Mean values from 10 assays \pm S.D.; $(\square)$ control veins; $(\square)$, pre-eclamptic veins; ${ }^{*} P<0.05$.

the vein and it was unchanged in pre-eclampsia (Bańkowski et al., 1996).

Umbilical cord vein walls were submitted to pepsin digestion and differential salt precipitation for isolation of particular collagen types. These were characterised by Western immunoblot analysis. Identification of each type of collagen was done by precipitation at specific $\mathrm{pH}$ conditions and $\mathrm{NaCl}$ concentrations, and immunoreaction with the respective anti-human monoclonal antibody after SDS/PAGE gel electrophoresis. Five collagen fractions were obtained from vein wall by this method. They showed typical electrophoretic patterns of types I, III, IV, V and VI collagen, 
like those described by Murata et al. (1986) and in our earlier papers (Bańkowski et al., 1993; 1996). Both the vein whole supernatant and the precipitated fractions containing type I and III collagen $\alpha$-subunits reacted with specific antibodies.

Type IV, V and VI collagen $\alpha$-subunits were visualised well only after differential salt precipitation because of their low content (not shown) and they penetrated the gel only after disulphide bonds reduction (Hudson et al., 1993; Kielty et al., 1990). No differences in electrophoretic mobility of collagen subunits were found between the control and preeclamptic veins.

It is known that type I collagen is the predominant species of this protein in normal adult human vein (Waksman et al., 1997) and this holds also for umbilical vein. The veins of pre-eclamptic newborns contain relatively less type I collagen and more type III collagen. The same differences were observed in pre-eclamptic UCA wall (Bańkowski et al., 1993). The pre-eclamptic Wharton's jelly was characterised by the increase in type III collagen content (Bańkowski et al., 1996). It can be concluded that pre-eclampsia is associated with an increase in type III collagen content in all umbilical cord tissues.

Another characteristic feature of these tissues is a decreased total collagen content and decreased its solubility. This phenomenon is probably connected with an increase in the degree of collagen polymerization. It is known that polymeric collagen becomes insoluble in neutral salts and in acidic solutions. The decrease of solubility may be also evoked by a relative increase of type III collagen content in veins of the pre-eclamptic newborns as it is well known that collagen of this type demonstrates lower solubility than type I collagen (Miller, 1976).

The presented changes in vein collagen of pre-eclamptic newborns are associated with extracellular matrix rearragement of the whole umbilical cord (Bańkowski, 1999; Bańkowski et al., 1993; 1996; Pawlicka et al.,
1999; Romanowicz et al., 1998; Romanowicz \& Sobolewski, 2000). It seems possible that similar alterations occurring in other fetal blood vessels may evoke an increase in peripheral resistance and result in an increase in the fetal blood pressure. The raised pressure may persist after birth (Griffin et al., 1983).

The newborns delivered by mothers with pre-eclampsia had a distinctly lower body mass than the controls. It is apparent from the reports of several authors that low birth weight may be a predictor of raised blood pressure in childhood and adult life (Baker et al., 1989; Gilligan \& Spector, 1984; Law et al., 1993; Whincup et al., 1995). It may be supposed that pre-eclampsia is a factor which evokes the initiation of hypertension in utero and its hightening throughout childhood and adult life.

\section{R E F E R E N C E S}

Aalkjaer C, Danielsen H, Johannsen P, Pedersen EB, Rasmussen A, Mulvany MJ. (1985) Abnormal vascular function and morphology in preeclampsia: a study of isolated resistance vessels. Clin Sci.; 69: 477-82.

Baker DJP, Osmond C, Golding J, Kuh D, Wadsworth MEJ. (1989) Growth in utero, blood pressure in childhood and adult life and mortality from cardiovascular diseases. Br Med J.; 298: 564-7.

Bańkowski E. (1999) Collagen of the umbilical cord and its alteration in EPH-gestosis (preeclapsia). Proc Ind Acad Sci (Chem Sci).; 111: 207-13.

Bańkowski E, Romanowicz L, Jaworski S. (1993) Collagen of umbilical cord arteries and its alterations in pre-eclampsia. $J$ Perinat Med.; 21: 491-8.

Bańkowski E, Sobolewski K, Romanowicz L, Chyczewski L, Jaworski S. (1996) Collagen and glycosaminoglycans of Wharton's jelly, and their alterations in pre-eclampsia. Eur $J$ Obstet Gynecol Reprod Biol.; 66: 109-17. 
Barnes MJ. (1983) Collagen polymorphism in the normal and diseased blood vessel wall. Atherosclerosis.; 46: 249-51.

Barnes MJ. (1985) Collagen in atherosclerosis. Collagen Rel Res.; 5: 65-97.

Brosens JA. (1977) Morphological changes in the utero-placental bed in pregnancy hypertension. Clin Obstet Gynec.; 4: 573-93.

Chung E, Miller E. (1974) Collagen polymorphism. Characterization of molecules with the chain composition $\alpha_{1}$ (III) in human tissues. Science.; 183: 1200-1.

Cretti A, Jaworski S. (1976) Studi istologici sulle arteriole miometrali di la fuori della sede placentare nella EPH gestosi. Patol Clin Ost.; 4: $225-36$.

Fox H. ed, (1997) Pathology of the placenta. pp 1-488, Saunders, London.

Gilligan JP, Spector S. (1984) Synthesis of collagen in cardiac and vascular walls. Hypertension.; 6: Suppl III 44-9.

Griffin D, Cohen-Overbeek T, Campbell S. (1983) Fetal and uteroplacental blood flow. Clin Obstet Gynecol.; 10: 565-02.

Howard RB. (1987) Control of human placental blood flow. Med Hypoth.; 23: 51-8.

Hudson BG, Reeders ST, Tryggvason K. (1993) Type IV collagen: structure, gen organization, and role in human diseases. $J$ Biol Chem.; 268: 26033-6.

Kielty CM, Boot-Handford RP, Ayad S, Shuttleworth CA, Grant ME. (1990) Molecular composition of type VI collagen. Evidence for chain heterogeneity in mammalian tissues and cultured cells. Biochem J.; 272: 787-95.

Kitzmiller JL, Bernirschke K. (1973) Immunofluorescent study of placental bed vessels in pre-eclampsia of pregnancy. Am J Obstet Gynecol.; 115: 248-51.

Laemmli UK. (1970) Cleavage of structural proteins during the assembly of the head of bacteriophage T4. Nature.; 227: 680-5.

Law CM, de Swiet M, Osmond C, Fayers PM, Barker DJ, Cruddas AM, Fall CH. (1993) Ini- tiation of hypertension in utero and its amplification throughout life. $\mathrm{Br}$ Med J.; 306: 24-7.

Miller EJ. (1976) Biochemical characteristics and biological significance of the genetically-distinct collagens. Mol Cell Biochem.; 13: $165-92$.

Murata K, Mitayama T, Kotake C. (1986) Changes of collagen types in various layer of the human aorta and their changes with the atherosclerotic process. Atherosclerosis.; 60: 251-62.

Pawlicka E, Bańkowski E, Jaworski S. (1999) Elastin of the umbilical cord arteries and its alterations in pre-eclampsia. Biol Neonate.; 75: 91-6.

Rippmann ET. (1981) Epidemiology of EPH-gestosis. In Current status of EPH-gestosis. Kurjak A, Rippmann ET, Sulovic V. eds, pp 100-120, Excerpta Medica, Amsterdam, Oxford, Princetown.

Romanowicz L, Bańkowski E, Jaworski S. (1998) Electrophoretic and chromatographic patterns of glycosaminoglycans of the umbilical cord vessels and their alterations in EPH-gestosis. Acta Biochim Polon.; 45: $805-9$.

Romanowicz L, Sobolewski K. (2000) Extracellular matrix components of the wall of umbilical cord vein and their alterations in pre-eclampsia. J Perinat Med.; 28: 140-6.

Schofield JD, Freeman IL, Jackson DS. (1971) The isolation and amino acid and carbohydrate composition of polymeric collagens prepared from various human tissues. Biochem J.; 124: 467-73.

Steven FS. (1967) The effect of chelating agents on collagen interfibrillar matrix interactions in connective tissue. Biochim Biophys Acta.; 140: $522-8$.

Van der Rest M, Garrone R. (1991) Collagen family of proteins. FASEB J.; 5: 2814-23.

Waksman Y, Mashiah A, Hod I, Rose SS, Friedman A. (1997) Collagen subtype pattern in normal and varicose saphenous veins in humans. Isr J Med Sci.; 33: 81-6. 
Whincup P, Cook D, Papacosta O, Walker M. (1995) Birth weight and blood pressure: cross sectional and longitudinal relations in childhood. Br Med J.; 311: 733-6.
Woessner Jr JF. (1976) Determination of hydroxyproline in connective tissue. In The methodology of connective tissue. Hall DA ed, pp 100-2. Joynson-Bruvers, Oxford. 